\title{
Das unbekannte Wesen
}

VON BERND HALFAR UND

ANDREA RABL

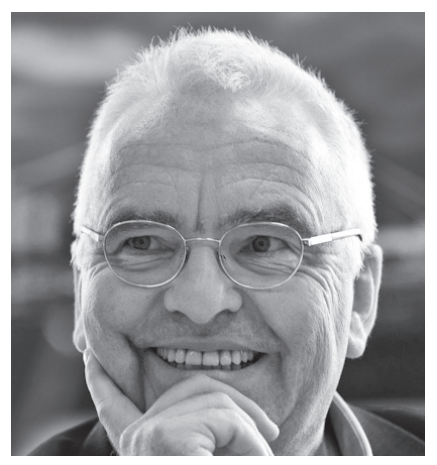

Prof. Dr. Bernd Halfar lehrt Management in sozialen Einrichtungen und Organisationsentwicklung an der Katholischen Universität Eichstätt-Ingolstadt. Er ist Mitgründer und Partner der Unternehmensberatung xit.forschen.planen.beraten $\mathrm{GmbH}$ mit Büros in Nürnberg und Berlin. E-Mail

bernd.halfar@ku-eichstaett.de

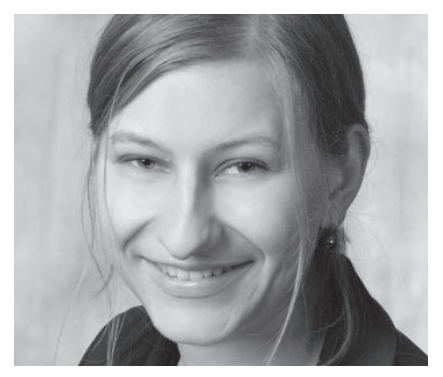

Andrea Rabl schrieb ihre Bachelorarbeit über Führungskräfte im Sozialbereich an der Arbeitsstelle NPO-Controlling an der Katholischen Universität EichstättIngolstadt mit Unterstützung der xit Gmbh. Sie arbeitet jetzt als Sozialpädagogin beim KolpingBildungswerk in Ingolstadt.

\author{
Über Führungskräfte in der Sozialwirtschaft gibt \\ es wenig empirisches Material. Eine Studie an \\ der Katholischen Universität Eichstätt-Ingolstadt \\ brachte nun zumindest etwas Licht ins Dunkel. Ein \\ Ergebnis: In sozialen Diensten und Einrichtungen \\ gibt es drei typische Karriereverläufe.
}

Welche Personen die Leitungspositionen im Bereich der Sozialwirtschaft besetzen - und damit das Bild der Sozialen Arbeit maßgeblich mitgestalten - ist weitgehend unbekannt.

Etwas Licht sollte eine Studie bringen, in der Führungskräfte bedeutender sozialer Einrichtungen im süddeutschen Raum zu ihren Karriereverläufen befragt wurden. Von 110 Personen, an die ein Fragebogen geschickt wurde, beteiligten sich 52 Probanden (Rücklaufquote $47 \%$ ), von denen 46 Männer $(90,2 \%)$ und 5 Frauen $(9,8 \%)$ im Alter zwischen 31 und 69 mit einem Durchschnittsalter von 55 Jahren waren.

Die Mehrheit der befragten Führungskräfte ordnete das eigene Elternhaus einer gehobenen sozialen Lage zu, aber ein beträchtlicher Teil (37,3\%) sah sein Elternhaus in der Arbeiterschicht verortet. Bestätigt wird dieser Befund durch die Angabe der Bildungsnähe des Elternhauses. So stellte sich heraus, dass ein großer Teil $(50,9 \%)$ der befragten Führungskräfte aus einem Elternhaus mit vergleichsweise geringer Bildungsnähe stammt.

Die soziale Herkunft bei Führungspersonen im Sozialbereich ist also gemischter, weniger sozial vererbt als in anderen Wirtschaftsbranchen. Auch Personen aus eher bildungsfernen Elternhäusern oder aus Elternhäusern einer vergleichsweise niedrigen sozialen Lage haben hier bessere Chancen, eine Spitzenposition zu erreichen.
Doch die in anderen Unternehmen immer wieder auffindbaren Aufstiegswege »vom Lehrling bis zum Vorstand « sind in sozialen Organisationen nicht feststellbar. Alle befragten Führungskräfte haben ein Hochschulstudium absolviert, einige im Anschluss ein Zweit- oder Aufbaustudium. 37 Prozent der Führungskräfte durchliefen zudem eine oder mehrere Berufsausbildungen vor ihrem Studium. Diese haben jedoch im Vergleich zu den gewählten Hochschulstudien eine geringe Bedeutung für die Karriereverläufe der Befragten. Die Mehrheit der befragten Leitungskräfte absolvierten theologische, wirtschaftswissenschaftliche oder pädagogischsoziale Studien. Auffällig ist der hohe Anteil der Führungskräfte (27,5\%), der diese Studienfächer miteinander kombinierte oder durch einen weiteren Qualifizierungsbereich ergänzte.

Je nach Studienfach ergeben sich aber Unterschiede im Ablauf der Karrierewege. Erkennbar sind drei idealtypische Verläufe:

- Zum einen fällt das kirchliche Muster auf, das häufig in Form einer »Blitzkarriere « - also mit einem sehr schnellen, plötzlichen Aufstieg - erfolgte. Diese »sprungfixe« Karriere kam insbesondere bei Personen vor, die Theologie studiert hatten.

- Ebenfalls gehäuft kam die wirtschaftlich geprägte, konventionelle Karriere mit stufenweisem Aufbau vor. Diese 


\section{Wer zählt eigentlich als Führungskraft?}

Eine Führungskraft ist eine Person, die Leitungsaufgaben in einer Organisation oder einem Unternehmen wahrnimmt, wobei es sich bei der Führung um eine (von mehreren) Managementfunktionen wie zum Beispiel Planung, Organisation, Führung und Kontrolle handelt. Die Definition von "Führungskraft « und "leitender Position « ist nicht einheitlich. Der Personenkreis muss daher, beispielsweise für Umfragen und Studien, jeweils definiert werden. In einer Studie des Vereins Deutscher Ingenieure werden Führungskräfte als "Personen mit Budget- und/oder Personalverantwortung " bezeichnet. Das Deutsche Institut für Wirtschaftsforschung definierte für ihren Führungskräfte-Monitor Führungskräfte als "Personen ab 18 Jahren, die im SOEP [Sozio-oekonomische Panel, eine seit 25 Jahren laufende repräsentative
Wiederholungsbefragung im Auftrag des Deutschen Instituts für Wirtschafts forschung] angaben, als Angestellte in der Privatwirtschaft in Funktionen mit umfassenden Führungsaufgaben [...], sonstigen Leitungsfunktionen oder hochqualifizierten Tätigkeiten [...] tätig zu sein«. Geschäftsführer und Vorstände werden oft auch juristisch definiert nämlich als gesetzlicher Vertreter und verantwortlicher Leiter, beispielsweise einer $\mathrm{GmbH}$, wobei der Umfang der Vertretungsmacht im "Gesetz betreffend die Gesellschaften mit beschränkter Haftung " (GmbHG) nicht ausdrücklich geregelt ist ( $\$ 35 \mathrm{ff}$.).

\section{Ouellen: Internet}

http://www.wikipedia.de, http://wirtschaftslexikon.gabler.de
Stufenkarriere ist typisch für Leitungspersonen, die eine wirtschaftswissenschaftliche Ausbildung vorweisen. Egal ob, so die Regel, die Karriere zunächst in einer anderen Branche mit anschließendem Wechsel in den sozialen Sektor begonnen wurde oder nach Studiumende direkt in der Sozialwirtschaft, verlief die Karriere stufenweise, mit einem kontinuierlichen Aufstieg in Positionen, die jeweils mit mehr Verantwortung verbunden waren als in der vorigen Position. $\mathrm{Zu}-$ dem waren die Tätigkeitsbereiche in diesen Karrieren mit kaum fachlicher Varianz wirtschaftlich geprägt.

- Führungskräfte, die eine sozialpädagogische Ausbildung haben, durchliefen in der Regel ebenfalls eine konventionelle Karriere mit stufenweisem Aufbau in sozialpädagogischen Aufgabenbereichen; allerdings starteten diese Karrieren fast immer direkt im sozialwirtschaftlichen Bereich.

Uns interessierten auch die von den Führungskräften als zentral eingeschätzten »Karrieretreiber«. An der Spitze der Skala werden von den Führungskräften vor allem ihre persönlichen Eigenschaften als entscheidend für ihren beruflichen Erfolg im Bereich der Sozialwirtschaft wahrgenommen. Auch die fachliche Qualifikation wird als wichtiger Karrierefaktor eingeschätzt. Als weniger bedeutend für den Karriereverlauf wur- den der Einfluss von Elternhaus, Auslandserfahrungen und der Studienort eingeordnet.

Bei dem Faktor »Fort- und Weiterbildungen« gab es eher skeptische Rückmeldungen für die Karriererelevanz. Lediglich ein kleinerer Teil der Führungskräfte gab an, dass sie ihre heutige Position ohne spezifische Fort- und Weiterbildungen nicht erreicht hätten. Insgesamt wurden Kenntnisse aus der Fort- und Weiterbildung als Karrierefaktor nur im mittleren Führungsbereich verortet.

Umso höher in der Hierarchie, desto prägnanter werden persönliche Eigenschaften als Karriereerklärung herangezogen. Als bedeutsam für eine Karriere im sozialen Sektor wurde von den Führungskräften das ehrenamtliche Engagement in der »Vorkarriere " sowie das Engagement im Beruf über die beruflichen Anforderungen hinaus, eingestuft. Auch das Studienfach wurde von den Befragten als wichtig eingeschätzt, wobei die sehr unterschiedlichen Studienfächer der Untersuchungsteilnehmer darauf hinweisen, dass die Wahl des Studienfaches auf die Karriere im sozialen Sektor nur bedingt Einfluss hat, wohl aber für die Karriereschneise innerhalb der Organisation.

Zumindest im »Ist-Bestand « der sozialwirtschaftlichen Führungskräfte lassen sich verschiedene Wege verfolgen, um in eine Führungsposition der Sozi- alwirtschaft zu gelangen. Das fachliche Know-how prägt die Wahrscheinlichkeit der Tätigkeit in der Führung, nicht aber die Wahrscheinlichkeit, eine Führungsposition zu erreichen. Diese Wahrscheinlichkeit scheint mit allgemeinen Eigenschaften von Führungskompetenz korreliert. Ob diese personellen Führungsstrukturen aus Sicht des Managements rational sind, bleibt eine Frage.

Im Moment liegen zu wenige Informationen vor, um beurteilen zu können, ob die vorgefundenen Rekrutierungsabläufe zu einer optimalen Besetzung der Führungspositionen im sozialen Sektor führen.

\section{Glosse}

\section{Management by Helikopter etc.}

Im Zuge des "Managements by Objectives" (Führen durch Zielvereinbarung) sind zahlreiche weitere Managementby-Konzepte formuliert worden. In der Führungspraxis werden die nachfolgenden Methoden oft angetroffen:

Management by Helikopter: über allem schweben, von Zeit zu Zeit auf den Boden zurückkommen, viel Staub aufwirbeln und dann wieder $a b$ in die Wolken

Management by Känguru: mit leerem Beutel große Sprünge machen

Management by Dübel: Lücke erkennen, schnell reinquetschen und sofort breitmachen

Management by Jeans: an allen entscheidenden Stellen sitzt eine Niete

Management by Champignon: die Mitarbeiter im Dunkeln lassen, mit Mist bewerfen, wenn die den Kopf herausstrecken: abschneiden

Management by Titanic: perfekt planen - und absaufen

Management by Robinson: alle warten auf Freitag

Management by Herodes: intensiv nach dem geeignetsten Nachfolger suchen und ihn dann feuern

Management by Nilpferd: auftauchen, Maul aufreißen, wieder abtauchen

Management by Pingpong: jeden Vorgang so lange hin- und herleiten, bis er sich erledigt hat

Management by Moses: das Volk in die Wüste schicken und auf Wunder warten 